\title{
Analyzing radio interferometric positioning systems with undersampling receivers
}

\author{
Marie Shinotsuka ${ }^{1 *}$, Yiyin Wang ${ }^{2}$, Xiaoli Ma ${ }^{1}$ and G. Tong Zhou ${ }^{1}$
}

\begin{abstract}
Radio interferometric positioning systems are developed for localization in wireless sensor networks (WSNs), and they have the potential to yield highly accurate location information at low computational cost and implementation complexity. In the radio interferometric positioning system (RIPS), two transmitters transmit sinusoidal signals at slightly different frequencies, and two receivers pass the received signals through square-law devices to produce low-frequency differential signals. However, a squaring operation increases the noise power, leading to performance loss. To avoid this problem, a receiver for the RIPS using undersampling techniques (RIPS-u) has been proposed. In this paper, we investigate the performance of the RIPS with a square-law device (RIPS-sq) and the performance of the RIPS-u through theoretical and experimental analyses. Specifically, we compute Cramér-Rao lower bounds (CRLBs) of the range and location estimates in both systems and show that the RIPS-u has lower CRLBs than the RIPS-sq. Furthermore, we have carried out experimental tests by implementing both systems on National Instruments (NI) Universal Software Radio Peripherals (USRPs). From both the theoretical and experimental results, the effectiveness of the RIPS-u over the RIPS-sq is confirmed.
\end{abstract}

Keywords: Wireless sensor network; Localization; Radio interferometric positioning system

\section{Introduction}

Advances in technology have enabled small-sized devices equipped with sensors to form wireless sensor networks (WSNs) [1]. These small devices act as nodes in WSNs, and their location information is a critical part of the sensor data [2]. Thus, localization has to be performed when node locations are unknown [3]. Global Positioning System (GPS) is a well-known example for localization. The global coverage and wide availability in commercial devices make GPS attractive. However, GPS requires a line-of-sight (LOS) path from the satellites in order to function, and this renders GPS inadequate for indoor scenarios. Furthermore, power consumption and computational costs of GPS are often prohibitively high for resource-limited WSNs [4]. For the same reason, ultrawideband (UWB)-based localization systems, which are known for high accuracy and robustness to multipath $[5,6]$, are also considered as unsuitable for WSNs [4]. Hence, the investigation of highly accurate localization

*Correspondence: gtg594x@mail.gatech.edu

1 School of Electrical and Computer Engineering, Georgia Institute of Technology, 30332-0250 Atlanta, GA, USA

Full list of author information is available at the end of the article systems using narrowband signals with low complexity is of great interest. One such system is the radio interferometric positioning system (RIPS) [7].

The RIPS is categorized as a range-based system, where the location is estimated from range measurements. The RIPS model to obtain range information is illustrated in Fig. 1. Each ranging session involves four nodes (two transmitters and two receivers); two transmitter nodes transmit pure sinusoidal signals at slightly different frequencies to produce slowly varying envelopes at the receivers. The range information is contained in the phases of the received signals, and the difference of the phases at two receivers yields a range metric called Qrange $q=d_{1,1}-d_{1,2}-d_{2,1}+d_{2,2}$, where $d_{k, m}$ is the distance between the $k$ th transmitter and the $m$ th receiver; (see Fig. 1 for illustration). After obtaining multiple Q-range measurements, node locations can be estimated. In $[7,8]$, several iterative approaches with genetic algorithm are used to collectively estimate the node locations in WSNs. Since iterative approach is computationally demanding, the computation is done at a centralized server. Another approach is a hyperbolic positioning method [9], which locates a single node at a time. In this

\section{是 Springer}

(c) 2015 Shinotsuka et al. Open Access This article is distributed under the terms of the Creative Commons Attribution 4.0 International License (http://creativecommons.org/licenses/by/4.0/), which permits unrestricted use, distribution, and reproduction in any medium, provided you give appropriate credit to the original author(s) and the source, provide a link to the Creative Commons license, and indicate if changes were made. 


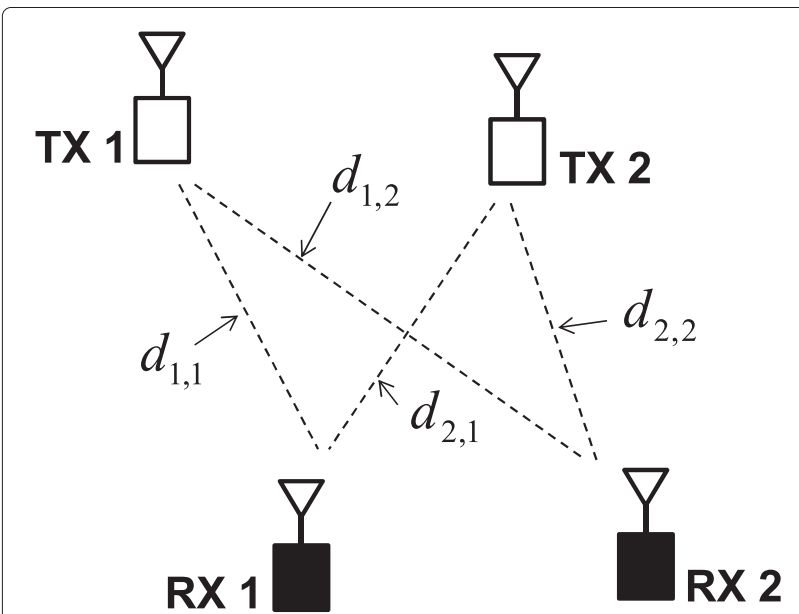

Fig. 1 The radio interferometric ranging technique

paper, we show that the Q-range can be converted to the range difference (RD), and the location can be estimated linearly when enough Q-range measurements are available.

In the original RIPS in [7], each receiver is equipped with a square-law device to extract a low-frequency differential signal. However, squaring the signal increases the noise power, which in turn deteriorates the rangeestimation performance. To avoid this problem, the RIPS with receivers using undersampling techniques (RIPS-u) is proposed in [10]. The transmitter model of the RIPS$\mathrm{u}$ is the same as the original RIPS with a square-law device (RIPS-sq), but the RIPS-u directly samples the signal without squaring operation. While the complexity of the RIPS- $u$ is reduced from that of the RIPS-sq by removing the square-law device, the results presented in [10] show that the RIPS- $u$ yields a 3-dB performance gain over the RIPS-sq, and thus the RIPS-u holds great promise.

In this paper, we investigate the performance of the RIPS- $u$ and the RIPS-sq through theoretical and experimental analyses to confirm that the RIPS- $u$ has better performance than the RIPS-sq. For the theoretical analysis, we compute Cramér-Rao lower bounds (CRLBs) for the Q-range and location estimates in both systems. We show that with least-squares (LS) estimators, the resulting performances approach the CRLBs. We have also implemented both systems using National Instruments (NI) Universal Software Radio Peripherals (USRPs) and further confirm the efficiency of the RIPS-u over the RIPS-sq.

The rest of this paper is organized as follows. An overview of the RIPS and related work are briefly discussed in Section 2. In Section 3, the system models of the RIPS- $u$ and the RIPS-sq are described. Their performances are analyzed theoretically by investigating the CRLBs of the Q-range estimates and the location estimates in Section 4. Simulation results are shown in
Section 5, and experimental results are presented in Section 6. Finally, conclusions are drawn in Section 7.

\subsection{Notations}

Bold uppercase letters denote matrices. Bold lowercase letters denote vectors. $\mathcal{C N}\left(\mu, \sigma^{2}\right)$ refers to a complex Gaussian distribution with mean $\mu$ and variance $\sigma^{2}$, and $\mathcal{U}(a, b)$ signifies a uniform distribution over the range $[a, b]$. Superscripts $(\cdot)^{\mathrm{T}},(\cdot)^{\mathrm{H}},(\cdot)^{*}$, and $(\cdot)^{\dagger}$ denote transpose, Hermitian transpose, complex conjugate, and pseudo-inverse, respectively.

\section{Related work}

Because of its flexibility and low complexity, the RIPS has been applied for various scenarios. One of which is to track mobile nodes [11-13]. In [11], a mobile node tracking system based on the RIPS called inTrack is proposed to analyze the effects of velocity and moderate outdoor multipath on the system performance. The system is further improved in [12] by incorporating a Doppler shift into location estimation. Their experimental results show a mean absolute error (MAE) of $37 \mathrm{~cm}$. Also taking a Doppler shift into account and using an extended Kalman filter, a tracking system based on the RIPS yields the MAE of $1.68 \mathrm{~m}$ in a field test [13]. Another extension of the RIPS is its implementation at a different frequency band. Formerly, the RIPS is implemented on CC1000 RF transceiver [14] at the frequency band below $1 \mathrm{GHz}$. However, in $[15,16]$, the RIPS is implemented on CC2430 transceivers [17], which operate at $2.4 \mathrm{GHz}$. Due to lack of finefrequency tuning capability of the CC2430 platform, an inherent offset of local oscillators is used for the frequency difference, resulting in the MAE of 1.5-2 $\mathrm{m}$ [15]. Using the same platform, a stochastic RIPS (SRIPS) [16] is proposed to improve the accuracy at $2.4 \mathrm{GHz}$ by taking into account some stochastic properties of Q-range measurements.

To use the RIPS for multipath-rich indoor environments, a multihop scheme is proposed in [18]. The approach is similar to [19], where sinusoids are transmitted at multiple frequencies, and a subspace-based method is used to detect the first-arriving path. On the other hand, the ranging signal of the RIPS is modified in [20-22] to make the system robust to the fading. A dual-tone RIPS (DRIPS) [20] uses dual-tone signaling to cancel the phase shifts due to fading. Synchronized anchor nodes transmit dual-tone signals simultaneously, which allow the target node to directly estimate its position. The DRIPS is further enhanced in uDRIPS [21] by employing undersampling techniques at the receiver. A space-time RIPS (STRIPS) [22] uses a space-time code and millimeter wave (MMW) band to combat fading and multipath effects. These aforementioned are some of the examples, and they show the flexibility of the RIPS. However, to the best of our knowledge, formal investigation of the performance 
bounds for the RIPS has not appeared in the literature. Our theoretical analysis presented in this paper can serve as benchmark to gauge various proposed algorithms.

\section{System model}

\subsection{Transmitter design}

A typical RIPS consists of two transmitters and two receivers in each ranging session. These nodes require perfect frequency synchronization. Time synchronization is required between two receivers, but the transmitters can be asynchronous. The transmitted signal at the $k$ th transmitter node in a band-pass complex form is represented as

$$
s_{k}(t)=a_{k} e^{j \theta_{k}} e^{j 2 \pi f_{k} t}, k=1,2,
$$

where $a_{k} e^{j \theta_{k}}$ and $f_{k}$ are the complex amplitude and the frequency, respectively, of the signal transmitted by the $k$ th node. Let us further define $\Delta=f_{1}-f_{2}$ and $g=\left(f_{1}+f_{2}\right) / 2$. We assume $f_{1}>f_{2}$ without loss of generality.

The block diagram of the RIPS- $u$ and the RIPS-sq is shown in Fig. 2. The received signal at the $m$ th receiver is modeled as

$$
\begin{aligned}
r_{m}(t) & =a_{1} e^{j 2 \pi\left(f_{1}-f_{o}\right) t} e^{-j \varphi_{1, m}}+a_{2} e^{j 2 \pi\left(f_{2}-f_{o}\right) t} e^{-j \varphi_{2, m}}+v_{m}(t), \\
m & =1,2,
\end{aligned}
$$

where $f_{o}$ is the frequency of the local oscillator that downconverts the signal to an intermediate frequency band, $\varphi_{k, m}=2 \pi f_{k}\left(d_{k, m} / c+t_{k}\right)-\theta_{k}$ is the phase of the received signal from the $k$ th transmitter to the $m$ th receiver, $d_{k, m}$ is the distance between the $k$ th transmitter and the $m$ th receiver, $c$ denotes the speed of light $\left(c=3 \times 10^{8} \mathrm{~m} / \mathrm{s}\right), t_{k}$ is the unknown time instant when the $k$ th node starts its transmission, and $v_{m}(t)$ is the additive noise. Although the RIPS works in the presence of stationary noise in general, for purposes of finding the CRLBs, we assume $v_{m}(t)$ to be white Gaussian noise denoted as $v_{m}(t) \sim \mathcal{C N}\left(0, \sigma_{m}^{2}\right)$ in this paper. In other words, the real and imaginary parts of $v_{m}(t)$ are assumed to be mutually independent real-valued white Gaussian processes with zero mean and variance $\sigma_{m}^{2} / 2$.

\subsection{Receiver design in the RIPS-sq}

In the RIPS-sq [7], the received signal $r_{m}(t)$ is passed through a band-pass filter (BPF) to remove the out-ofband noise. Then, a square-law device squares the output of the BPF, and a low-pass filter (LPF) removes highfrequency components beyond $\Delta$. The output of the LPF is a low-frequency differential signal $\widetilde{r}_{m}(t)$, which only contains the frequency components at $\pm \Delta$. Sampling $\widetilde{r}_{m}(t)$ at the rate $f_{s} \geq 2 \Delta$, we obtain

$$
\widetilde{r}_{m}[n]=a_{1} a_{2} e^{j 2 \pi \Delta n / f_{s}-j \phi_{m}}+a_{1} a_{2} e^{-j 2 \pi \Delta n / f_{s}+j \phi_{m}}+\widetilde{v}_{m}[n],
$$

where $\phi_{m}=\varphi_{1, m}-\varphi_{2, m}=2 \pi f_{1}\left(d_{1, m} / c+t_{1}\right)-\theta_{1}-$ $2 \pi f_{2}\left(d_{2, m} / c+t_{2}\right)+\theta_{2}$, and $\widetilde{v}_{m}[n]$ is the sampled aggregated noise, which includes a noise product term and signal-noise product terms. Mathematically, $\widetilde{v}_{m}[n]$ can be written as

$$
\begin{aligned}
\widetilde{v}_{m}[n]= & v_{m}^{*}[n] \sum_{k=1}^{2} a_{k} e^{j 2 \pi\left(f_{k}-f_{o}\right) n / f_{s}-j \varphi_{k, m}} \\
& +v_{m}[n] \sum_{k=1}^{2} a_{k} e^{-j 2 \pi\left(f_{k}-f_{o}\right) n / f_{s}+j \varphi_{k, m}}+v_{m}[n] v_{m}^{*}[n] .
\end{aligned}
$$

Statistics of $\widetilde{v}_{m}[n]$ are investigated in Section 4.1. Collecting $N$ samples and stacking them vertically, (3) can be represented in a matrix-vector form as

$$
\widetilde{\mathbf{r}}_{m}=\widetilde{\mathbf{H}} \widetilde{\mathbf{z}}_{m}+\widetilde{\mathbf{v}}_{m}
$$

where $\widetilde{\mathbf{r}}_{m}$ and $\widetilde{\mathbf{v}}_{m}$ are column vectors of samples from $\widetilde{r}_{m}(t)$ and $\widetilde{v}_{m}(t)$, respectively, $\widetilde{\mathbf{H}}=\left[\mathbf{h}\left(\Delta, f_{s}\right), \mathbf{h}\left(-\Delta, f_{s}\right)\right]$, $\mathbf{h}\left(f, f_{s}\right)=\left[1, e^{j 2 \pi f / f_{s}}, \ldots, e^{j 2 \pi(N-1) f / f_{s}}\right]^{T}$, and $\widetilde{\mathbf{z}}_{m}=$ $\left[a_{1} a_{2} e^{-j \phi_{m}}, a_{1} a_{2} e^{j \phi_{m}}\right]^{T}$.

Notice that the range information is in the phase of $\widetilde{\mathbf{z}}_{m}$. To estimate the Q-range, the following assumptions are

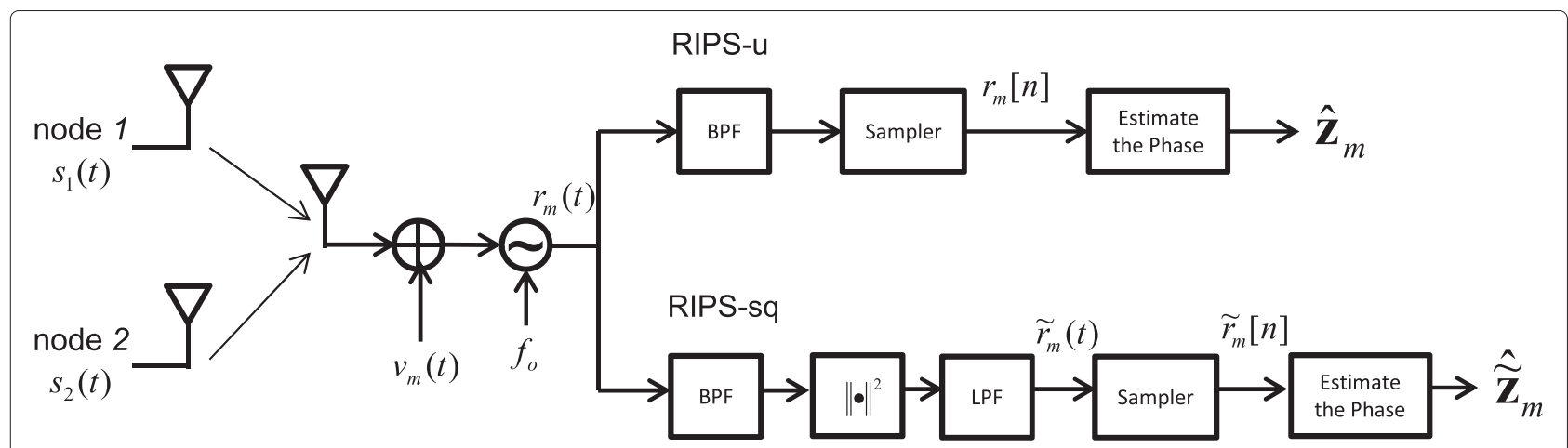

Fig. 2 The block diagram of RIPS-u and RIPS-sq 
made [7]: (1) $\Delta \ll g$, i.e., $f_{1}-f_{2} \ll\left(f_{1}+f_{2}\right) / 2$; (2) $|q|<c / 2 g$, where $q=d_{1,1}-d_{1,2}-d_{2,1}+d_{2,2}$ is the Q-range. From these assumptions, the phase difference at two receivers can be approximated as [7]

$$
\frac{c}{2 \pi g}\left(\phi_{1}-\phi_{2}\right)=q+\eta \approx q,
$$

where $\eta=\frac{\Delta}{4 \pi g}\left(d_{1,1}-d_{1,2}+d_{2,1}-d_{2,2}\right)$. Hence, the Qrange is estimated in the RIPS-sq as [7]

$$
\widehat{q}^{(\mathrm{sq})}=\frac{c}{2 \pi g}\left(\widehat{\phi}_{1}-\widehat{\phi}_{2}\right),
$$

where $\widehat{\phi}_{m}$ is the estimated phase at the $m$ th receiver. One way to estimate the phase is by using an LS estimator. Assuming the frequencies are known at the receivers, $\phi_{m}$ can be estimated as

$$
\widehat{\phi}_{m}=\arg \left(\left[\widehat{\mathbf{z}}_{m}\right]_{1}^{*}+\left[\widehat{\mathbf{z}}_{m}\right]_{2}\right),
$$

where $\widehat{\mathbf{z}}_{m}=\widetilde{\mathbf{H}}^{\dagger} \widetilde{\mathbf{r}}_{m}$ and $[\mathbf{z}]_{i}$ denotes the $i$ th element of the vector $\mathbf{z}$.

From (6), it is apparent that as $\Delta$ increases, $\eta$ becomes a dominant source of error in the range estimation of the RIPS-sq. Moreover, since the range is estimated from the phase, an unknown integer is present when $|q|>c / 2 g$, which results in integer ambiguity. In this paper, we call the maximum range that causes no integer ambiguity as a resolvable range. Here, the resolvable range in the RIPS-sq is $c / 2 g$.

In practice, the Q-range is likely to be larger than the resolvable range, and multiple Q-range measurements at different frequencies are obtained to resolve the integer ambiguity [7]. The unknown integers can be calculated with the maximum likelihood estimator (MLE) [23], the Chinese remainder theorem (CRT) $[24,25]$, or lattice reduction method [26]. In this paper, we ignore the integer ambiguity for simplicity and choose the parameters for simulations and experiments to avoid ambiguous measurements.

\subsection{Receiver design in the RIPS-u}

In the RIPS-u [10], the received signal $r_{m}(t)$ in (2) is passed through a BPF and directly sampled at the rate $f_{u}$, i.e.,

$$
\begin{aligned}
r_{m}[n]= & a_{1} e^{j 2 \pi\left(f_{1}-f_{o}\right) n / f_{u}} e^{-j \varphi_{1, m}}+a_{2} e^{j 2 \pi\left(f_{2}-f_{o}\right) n / f_{u}} e^{-j \varphi_{2, m}} \\
& +v_{m}[n] .
\end{aligned}
$$

The receiver design of the RIPS- $u$ is illustrated in the upper half of Fig. 2. At the $m$ th receiver, $N$ samples are collected, which are modeled as

$$
\mathbf{r}_{m}=\mathbf{H z}_{m}+\mathbf{v}_{m}
$$

where $\mathbf{r}_{m}$ and $\mathbf{v}_{m}$ are the sampled vectors of $r_{m}[n]$ and $v_{m}[n]$, respectively, $\mathbf{H}=\left[\mathbf{h}\left(f_{1}-f_{o}, f_{u}\right) \mathbf{h}\left(f_{2}-f_{o}, f_{u}\right)\right]$, and $\mathbf{z}_{m}=\left[a_{1} e^{-j \varphi_{1, m}}, a_{2} e^{-j \varphi_{2, m}}\right]^{T}$. As shown in [10], even $f_{u}<2\left(f_{k}-f_{o}\right)$, where the frequencies are aliased, the RIPS-u can still accurately estimate the phase as long as the aliased frequencies are well separated. In other words, the frequencies are to be designed to satisfy the condition such that $\bmod \left(\Delta, f_{u}\right) \neq 0$ to prevent frequencies from aliasing onto each other, where $\bmod (\Delta, f)=\Delta-$ $f\lfloor\Delta / f\rfloor$ with $\lfloor\cdot\rfloor$ denoting the floor function.

Assuming the knowledge of designed parameters is shared among all the participating nodes, the LS estimator for the phase vector $\mathbf{z}_{m}$ is

$$
\widehat{\mathbf{z}}_{m}=\mathbf{H}^{\dagger} \mathbf{r}_{m} \text {. }
$$

When $\left|d_{1,1}-d_{1,2}\right|<c /\left(2 f_{1}\right)$ and $\left|d_{2,1}-d_{2,2}\right|<c /\left(2 f_{2}\right)$, no unknown integers are generated from phase unwrapping. In such a case, a Q-range estimator of the RIPS- $u$ is given as

$$
\begin{aligned}
\widehat{q}^{(u)} & =\frac{c}{2 \pi}\left\{\frac{1}{f_{1}} \arg \left(\left[\widehat{\mathbf{z}}_{1}\right]_{1}^{*}\left[\widehat{\mathbf{z}}_{2}\right]_{1}\right)+\frac{1}{f_{2}} \arg \left(\left[\widehat{\mathbf{z}}_{1}\right]_{2}\left[\widehat{\mathbf{z}}_{2}\right]_{2}^{*}\right)\right\} \\
& =\frac{c}{2 \pi}\left(\frac{\widehat{\varphi}_{1,1}-\widehat{\varphi}_{1,2}}{f_{1}}-\frac{\widehat{\varphi}_{2,1}-\widehat{\varphi}_{2,2}}{f_{2}}\right) .
\end{aligned}
$$

Notice that the formation of the estimator in (12) does not involve any approximation in contrast to (7) for the RIPS-sq. Moreover, the RIPS-sq requires the frequencies to satisfy $\Delta \ll f_{1}, f_{2}$ to attain a small approximation error and to guarantee a slowly varying envelope of the received signal. Such a requirement is not imposed in the RIPS-u, and thus, the RIPS- $u$ has wider applicability.

Unfortunately, neither the RIPS-u nor the RIPS-sq is robust to multipath [27]. This is because the fading channel causes unknown phase shifts in the received signal. As four nodes are spatially apart, four links in Fig. 1 have independent channels. Hence, the phase shifts due to multipath fading cannot be canceled out with the Qrange estimators of both systems, resulting in the biased Q-range estimates. To effectively combat the multipath fading effects, the methods [20-22] discussed in Section 2 should be considered.

\subsection{Localization}

In the original RIPS [7], locations of the nodes in the WSN are estimated collectively. The iterative algorithm used in [7] is computationally expensive, and a dedicated server is required to perform the localization. However, by restricting the Q-range measurements to have one unknown node, localization can be performed at lower complexity. The assumption we made here is applicable when we have enough anchor nodes and one target node or localizing one node at a time [12].

When there exists only one node with unknown position in a ranging session, the Q-range can be converted 
to the range difference (RD). For example in Fig. 1, let us assume that TX2, RX1, and RX2 are at known positions, and TX1 is the target node to be localized. Then, the last two terms in the Q-range $q=d_{1,1}-d_{1,2}-d_{2,1}+d_{2,2}$ can be pre-calculated. Moving the unknown terms to the left-hand side (LHS), we arrive at the RD measurement between TX1 and two receivers as

$$
d_{1,1}-d_{1,2}=q+d_{2,1}-d_{2,2}
$$

Let us denote the coordinates of the target node and the $m$ th receiver as $\mathbf{x}$ and $\mathbf{x}_{m}$, respectively. Choosing the first receiver node as the origin of the coordinate system $\left(\mathbf{x}_{1}=\right.$ 0), (13) can be rewritten as

$$
\|\mathbf{x}\|-\left\|\mathbf{x}-\mathbf{x}_{2}\right\|=R,
$$

where $R$ is the right-hand side (RHS) of (13) and $\|\mathbf{x}\|$ denotes the Euclidean norm of the vector $\mathbf{x}$. Rearranging the terms and squaring both sides as

$$
\left\|\mathbf{x}-\mathbf{x}_{2}\right\|^{2}=(\|\mathbf{x}\|-R)^{2},
$$

we arrive at

$$
2 R\|\mathbf{x}\|-2 \mathbf{x}_{2}^{T} \mathbf{x}=R^{2}-\left\|\mathbf{x}_{2}\right\|^{2},
$$

where the unknown terms are collected on the LHS, and the known terms are on the RHS. When we consider a $2 \mathrm{D}$ scenario, the dimension of $\mathbf{x}$ is 2 . Keeping $\mathbf{x}_{1}$ fixed as the reference node over multiple RD measurements, we have $\mathbf{x}$ and $\|\mathbf{x}\|$ as unknowns. Hence, with at least three independent RD measurements, we have a set of linear equations to solve the location vector $\mathbf{x}$. Details of RDbased localization algorithms with linear estimators can be found in $[28,29]$.

\section{Performance analysis}

In this section, we derive the CRLB for both the RIPS- $\mathrm{u}$ and the RIPS-sq based on the system models described in Section 3. We first compute the CRLBs of $\phi_{m}$ and $\varphi_{k, m}$ for the RIPS-sq and the RIPS- $u$, respectively, and perform the vector transformation to achieve the CRLBs of the Qrange estimates. In the following analysis, we assume that the Q-range is within the resolvable range for both systems, and thus we do not consider the integer ambiguity issue.

\subsection{The CRLB of the Q-range estimate in the RIPS-sq}

To derive the CRLB in the RIPS-sq, the statistical properties of the aggregated noise $\widetilde{v}_{m}[n]$ are analyzed first. Since $v_{1}[n]$ and $v_{2}[n]$ at a given time instant $n$ are treated as mutually independent Gaussian random variables $\left(v_{m}[n] \sim \mathcal{C N}\left(0, \sigma_{m}^{2}\right)\right)$ and the signal is squared at each receiver independently, the aggregated noise at each receiver $\widetilde{v}_{1}[n]$ and $\widetilde{v}_{2}[n]$ are also mutually independent.
Thus, the first- and second-order statistics of $\widetilde{v}_{m}[n]$ are given as [10]

$$
\begin{aligned}
E\left[\widetilde{v}_{m}[n]\right] & =\sigma_{m}^{2}, \\
\operatorname{var}\left[\widetilde{v}_{m}[n]\right] & =\sigma_{m}^{4}+2 \sigma_{m}^{2}\left\|u_{m}[n]\right\|^{2}, \\
\widetilde{\sigma}_{m}^{2}[n] & \triangleq \operatorname{var}\left[\widetilde{v}_{m}[n]\right],
\end{aligned}
$$

where $u_{m}[n]=\sum_{k=1}^{2} a_{k} e^{j 2 \pi\left(f_{k}-f_{o}\right) n / f_{s}} e^{-j \varphi_{k, m}}$ and recall $\varphi_{k, m}=2 \pi f_{k}\left(d_{k, m} / c+t_{k}\right)-\theta_{k}$. Notice that the noise variance depends on $n$. Hence, let us further approximate $\widetilde{\sigma}_{m}[n]$ by its mean over the random parameter $\theta_{k} \sim$ $\mathcal{U}(-\pi / 2, \pi / 2)$ in $\varphi_{k, m}$ as

$$
\bar{\sigma}_{m}^{2}=E_{\theta_{k}}\left[\tilde{\sigma}_{m}^{2}[n]\right]=\sigma_{m}^{4}+2 \sigma_{m}^{2}\left(a_{1}^{2}+a_{2}^{2}\right) .
$$

The exact distribution of the aggregated noise is difficult to derive. Instead, we use the approximation in (20) and model the aggregated noise as a normally distributed random variable such that $\widetilde{v}_{m}[n] \sim \mathcal{N}\left(\sigma_{m}^{2}, \bar{\sigma}_{m}^{2}\right)$. Later in this paper, we show that the Monte Carlo simulation results match well with the CRLB derived based on the Gaussian approximation, and thus the Gaussian approximation yields meaningful results.

The interest here is to find the CRLB of $\phi_{m}$. The lowfrequency differential signal in (3) can be equivalently written as a real sinusoid with an additive noise as

$$
\tilde{r}_{m}[n]=2 a_{1} a_{2} \cos \left(\frac{2 \pi \Delta n}{f_{s}}-\phi_{m}\right)+\widetilde{v}_{m}[n] .
$$

Since we model $\widetilde{v}_{m}[n]$ as Gaussian, the log-likelihood function for $\widetilde{\mathbf{r}}_{m}$ is represented as

$$
\begin{aligned}
\ln p\left(\widetilde{\mathbf{r}}_{m} ; \phi_{m}\right)= & -\frac{N}{2} \ln \left(2 \pi \bar{\sigma}_{m}^{2}\right)-\frac{1}{2 \bar{\sigma}_{m}^{2}} \sum_{n=0}^{N-1}\left(\widetilde{r}_{m}[n]\right. \\
& \left.-2 a_{1} a_{2} \cos \left(\frac{2 \pi \Delta n}{f_{s}}-\phi_{m}\right)-\sigma_{m}^{2}\right)^{2} .
\end{aligned}
$$

Taking a second derivative of (22) w.r.t. $\phi_{m}$, we obtain

$$
\begin{aligned}
\frac{\partial^{2} \ln p\left(\tilde{\mathbf{r}}_{m} ; \phi_{m}\right)}{\partial \phi_{m}^{2}}= & -\left(\frac{2 a_{1} a_{2}}{\bar{\sigma}_{m}}\right)^{2} \sum_{n=0}^{N-1}\left\{\sin ^{2}\left(\frac{2 \pi \Delta n}{f_{s}}-\phi_{m}\right)\right. \\
& -\cos ^{2}\left(\frac{2 \pi \Delta n}{f_{s}}-\phi_{m}\right)+\frac{1}{2 a_{1} a_{2}} \widetilde{r}_{m}[n] \\
& \times \cos \left(\frac{2 \pi \Delta n}{f_{s}}-\phi_{m}\right)-\frac{\sigma_{m}^{2}}{2 a_{1} a_{2}} \\
& \left.\times \cos \left(\frac{2 \pi \Delta n}{f_{s}}-\phi_{m}\right)\right\} .
\end{aligned}
$$

To compute the CRLB of $\phi_{m}$, we need to take the expectation of (23). Notice that $E\left[\widetilde{r}_{m}[n]\right]=$ $2 a_{1} a_{2} \cos \left(\frac{2 \pi \Delta n}{f_{s}}-\phi_{m}\right)+\sigma_{m}^{2}$, where the second term is due to the non-zero mean of $\widetilde{v}_{m}[n]$ as shown in (19). 
Hence, using the trigonometric identities, the CRLB of $\phi_{m}$ becomes

$$
\begin{aligned}
\operatorname{CRLB}\left(\phi_{m}\right) & =-\left(E\left[\frac{\partial^{2} \ln p\left(\widetilde{\mathbf{r}}_{m} ; \phi_{m}\right)}{\partial \phi_{m}^{2}}\right]\right)^{-1} \\
& =2\left(\frac{\bar{\sigma}_{m}}{2 a_{1} a_{2}}\right)^{2}\left\{N-\sum_{n=0}^{N-1} \cos \left(\frac{4 \pi \Delta n}{f_{s}}-2 \phi_{m}\right)\right\}^{-1} .
\end{aligned}
$$

Recall that in the RIPS-sq, the signal is sampled at the rate $f_{s}>2 \Delta$. Hence, we assume that $\bmod \left(2 \Delta, f_{s}\right)$ is not near 0 or 0.5 , which yields the approximations $\sum_{n=0}^{N-1} \cos \left(\frac{4 \pi \Delta n}{f_{s}}-2 \phi_{m}\right) \approx 0$ ([30] pp. 56), and the CRLB in (24) becomes

$$
\operatorname{CRLB}\left(\phi_{m}\right) \approx \frac{2}{N}\left(\frac{\bar{\sigma}_{m}}{2 a_{1} a_{2}}\right)^{2}
$$

From (7), in the RIPS-sq, the Q-range is approximated as $\widehat{q}^{(\mathrm{sq})} \approx \frac{c}{2 \pi g}\left(\widehat{\phi}_{1}-\widehat{\phi}_{2}\right)$ given $\Delta \ll g$. By performing the vector transformation, the CRLB of the Q-range estimate in the RIPS-sq is calculated as

$$
\begin{aligned}
\operatorname{CRLB}\left(q^{(\mathrm{sq})}\right) & =\left(\frac{c}{2 \pi g}\right)^{2}\left(\operatorname{CRLB}\left(\phi_{1}\right)+\operatorname{CRLB}\left(\phi_{2}\right)\right) \\
& =\left(\frac{c}{2 \pi}\right)^{2} \frac{\bar{\sigma}_{1}^{2}+\bar{\sigma}_{2}^{2}}{2 N}\left(\frac{1}{a_{1} a_{2} g}\right)^{2} .
\end{aligned}
$$

Notice that the CRLB depends on $\bar{\sigma}_{m}^{2}, a_{k}, g$, and $N$. According to (26), the CRLB decreases as the noise variance $\bar{\sigma}_{m}^{2}$ decreases, and $N$ and $g$ increase. Note, however, when transmitters transmit with high frequencies, the resolvable range becomes small, and the Q-range estimates are likely to experience the integer ambiguity problem.

\subsection{The CRLB of the Q-range estimate in the RIPS-u}

The CRLB of the Q-range estimate in the RIPS- $u$ is derived based on the system model in (10). Since the noise variance is independent of the parameter $\varphi_{k, m}$, the Fisher information matrix (FIM) $\mathbf{J}$ for the parameter vector $\boldsymbol{\varphi}_{m}=\left[\varphi_{1, m}, \varphi_{2, m}\right]^{T}$ under the complex Gaussian noise is expressed as ([30] pp. 525)

$$
\left[\mathbf{J}\left(\boldsymbol{\varphi}_{m}\right)\right]_{k l}=\frac{1}{\sigma_{m}^{2}} \dot{\mathbf{h}}\left(\varphi_{k, m}\right)^{\mathrm{H}} \dot{\mathbf{h}}\left(\varphi_{l, m}\right)
$$

where $[\mathbf{J}]_{k l}$ denotes the element in the matrix $\mathbf{J}$ at the $k$ th row and the $l$ th column and

$$
\dot{\mathbf{h}}\left(\varphi_{k, m}\right)=\left[\begin{array}{c}
\mathbf{h}\left(f_{k}-f_{o}, f_{u}\right)(-j) a_{k} e^{-j \varphi_{k, m}} \\
\mathbf{h}^{*}\left(f_{k}-f_{o}, f_{u}\right) j a_{k} e^{j \varphi_{k, m}}
\end{array}\right] .
$$

Substituting (28) into (27), we arrive at

$$
\mathbf{J}\left(\boldsymbol{\varphi}_{m}\right)=2\left(\frac{1}{\sigma_{m}}\right)^{2}\left[\begin{array}{cc}
a_{1}^{2} N & a_{1} a_{2} \sum_{n=0}^{N-1} \cos \left(\frac{2 \pi \Delta n}{f_{u}}-\phi_{m}\right) \\
a_{1} a_{2} \sum_{n=0}^{N-1} \cos \left(\frac{2 \pi \Delta n}{f_{u}}-\phi_{m}\right) & a_{2}^{2} N
\end{array}\right] .
$$

Taking the inverse of $\mathbf{J}\left(\boldsymbol{\varphi}_{m}\right)$, the CRLB of $\varphi_{k, m}$ is achieved along the diagonal as

$$
\operatorname{CRLB}\left(\varphi_{k, m}\right)=\frac{N}{2}\left(\frac{\sigma_{m}}{a_{k}}\right)^{2}\left\{N^{2}-\left(\sum_{n=0}^{N-1} \cos \left(\frac{2 \pi \Delta n}{f_{u}}-\phi_{m}\right)\right)^{2}\right\}^{-1} .
$$

Recall that to avoid closely aliased frequencies, the parameters are chosen so that $\bmod \left(\Delta, f_{u}\right) \neq 0$. Therefore, similarly to the approximation that we made in the RIPS-sq, we assume that $\sum_{n=0}^{N-1} \cos \left(\frac{2 \pi \Delta n}{f_{u}}-\phi_{m}\right) \approx 0$ and further simplify the CRLB in (30) as

$$
\operatorname{CRLB}\left(\varphi_{k, m}\right) \approx\left(\frac{\sigma_{m}}{a_{k}}\right)^{2} \frac{1}{2 N} .
$$

Sequentially, the CRLB of the Q-range for the RIPS$\mathrm{u}$ is calculated from the CRLBs of $\varphi_{k, m}$ given in (31). According to (12), the Q-range is calculated by $\widehat{q}^{(u)}=\frac{c}{2 \pi}\left(\frac{\widehat{\varphi}_{1,1}-\widehat{\varphi}_{1,2}}{f_{1}}-\frac{\widehat{\varphi}_{2,1}-\widehat{\varphi}_{2,2}}{f_{2}}\right)$. Hence, following the same transformation process as in the RIPS-sq, the CRLB of the Q-range for the RIPS- $u$ is obtained from the CRLB of $\varphi_{k, m}$ as

$$
\begin{aligned}
\operatorname{CRLB}\left(q^{(u)}\right) & =\sum_{k=1}^{2} \sum_{m=1}^{2}\left(\frac{c}{2 \pi f_{k}}\right)^{2} \operatorname{CRLB}\left(\varphi_{k, m}\right) \\
& =\left(\frac{c}{2 \pi}\right)^{2} \frac{\sigma_{1}^{2}+\sigma_{2}^{2}}{2 N}\left(\frac{1}{a_{1}^{2} f_{1}^{2}}+\frac{1}{a_{2}^{2} f_{2}^{2}}\right) .
\end{aligned}
$$

The CRLB of the Q-range depends on $\sigma_{m}^{2}, a_{k}^{2}, N$, and $f_{k}$. Now, let us investigate how different parameters affect the CRLBs in both systems. For simplicity, consider the special case where $\sigma_{m}^{2}=\sigma^{2}$ and $a_{1}=a_{2}=a$. In such a case, the CRLB of the Q-range estimate with the RIPS-sq in (26) becomes

$$
\gamma^{(\mathrm{sq})}=\left(\frac{c}{2 \pi}\right)^{2} \frac{\sigma^{2}\left(\sigma^{2}+4 a^{2}\right)}{a^{4} N}\left(\frac{1}{g^{2}}\right) .
$$

Similarly, the CRLB of the Q-range estimate with the RIPS-u in (32) becomes

$$
\gamma^{(u)}=\left(\frac{c}{2 \pi}\right)^{2} \frac{\sigma^{2}}{a^{2} N}\left(\frac{1}{f_{1}^{2}}+\frac{1}{f_{2}^{2}}\right) .
$$

Under this simplified scenario, the CRLBs of the Qrange estimates in both systems vs. signal-to-noise ratio (SNR) are plotted in Fig. 3. Here, we define the SNR at the $m$ th receiver as $\left(a_{1}^{2}+a_{2}^{2}\right) /\left(\operatorname{PSD}_{v_{m}}\left(f_{1}\right)+\operatorname{PSD}_{v_{m}}\left(f_{2}\right)\right)$, 


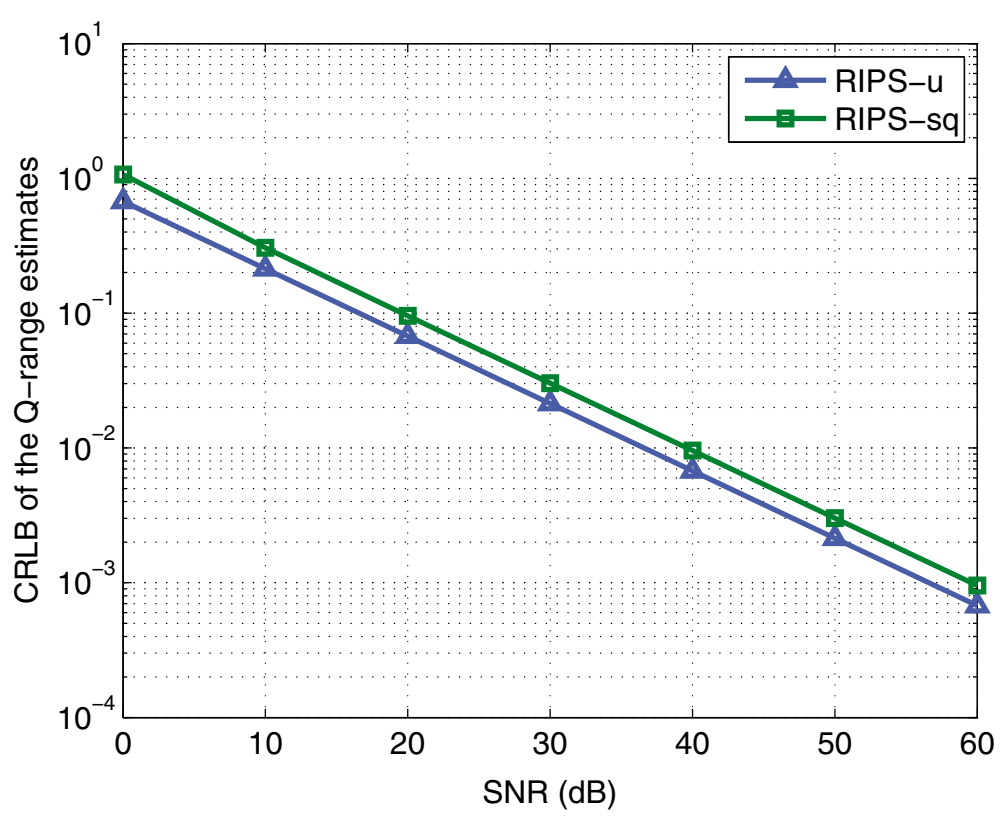

Fig. 3 The CRLB of the Q-range estimates vs. SNR

where $\operatorname{PSD}_{v_{m}}(f)$ denotes a power spectral density of the noise $v_{m}(t)$ at frequency $f$. Since we model the noise as Gaussian distributed with variance $\sigma_{m}^{2}=\sigma^{2}$ for $m=1,2$, $\operatorname{PSD}_{v_{m}}\left(f_{1}\right)=\operatorname{PSD}_{v_{m}}\left(f_{2}\right)=\sigma^{2}$, and the SNR is $a^{2} / \sigma^{2}$. For illustration purposes here, we choose $g=10 \mathrm{MHz}$, $\Delta=1.2 \mathrm{kHz}, f_{o}=9 \mathrm{MHz}$, and $N=100$. The CRLBs for both systems decrease with increasing SNR (a decreasing $\sigma^{2}$ ) as expected. Moreover, notice the 3 -dB gap between two curves. This is because when $\sigma^{2} \ll a^{2}$, the CRLB of the RIPS-sq in (33) is approximated as

$$
\gamma^{(\mathrm{sq})} \approx\left(\frac{c}{2 \pi}\right)^{2} \frac{4 \sigma^{2}}{a^{2} N g^{2}}
$$

Also, assuming $\Delta \ll g$ such that $f_{1} \approx f_{2} \approx g$, (34) becomes

$$
\gamma^{(u)} \approx\left(\frac{c}{2 \pi}\right)^{2} \frac{2 \sigma^{2}}{a^{2} N g^{2}} \approx 0.5 \gamma^{(\mathrm{sq})} .
$$

Hence, the RIPS- $u$ achieves a 3-dB gain over the RIPS-sq.

When the SNR is fixed at $30 \mathrm{~dB}$ and $g$ is varied $(\Delta$ is fixed at $1.2 \mathrm{kHz}$ ), the CRLB also decreases as shown in Fig. 4. However, recall that frequencies affect the resolvable range. In other words, when the signals are transmitted at a higher frequency band, the performance improves at the cost of a reduction in the resolvable range. The effect of $\Delta$ on the CRLB with fixed $g$ is shown in Fig. 5. Both CRLB curves are flat since the CRLB of the RIPSsq in (33) is independent of $\Delta$ and the change in $\Delta$ is small compared to $g$ for the RIPS-u in (34). Yet, a small $\Delta$ is desirable for the RIPS-sq since the approximated term $\eta$ depends on the the ratio $\Delta / g$. When $\Delta$ is large with respect to $g, \eta$ becomes a dominant source of error in the RIPS-sq.

Let us further analyze how signal amplitudes influence the performance. Constraining the total transmitting power as $a_{1}^{2}+a_{2}^{2}=1$ and fixing the SNR at $30 \mathrm{~dB}$, we plot the CRLB vs. $a_{1}^{2}$ in Fig. 6 . The performance varies with different $a_{1}^{2}$, and the minimum CRLB is attained when $a_{1}^{2}=a_{2}^{2}$ in both the RIPS- $\mathrm{u}$ and the RIPS-sq. Hence, equal transmitting power at two transmitters yields the best ranging performance in both systems.

\subsection{The CRLB of the location estimates}

Using the CRLBs for the Q-ranges derived in the previous subsections, the CRLBs of the location estimates in the RIPS- $u$ and the RIPS-sq are derived. As we have described in Section 3, we assume that positions of three nodes are known at each ranging session. In the following derivations, since both systems require time synchronization between receivers, we fix the target node as the first transmitter. Considering a 2D scenario, we denote the coordinates of the target node and the $m$ th receiver node as $\mathbf{x}=[x, y]^{\mathrm{T}}$ and $\mathbf{x}_{m}=\left[x_{m}, y_{m}\right]^{\mathrm{T}}$, respectively. Although we only consider a $2 \mathrm{D}$ scenario here, the same derivation can be applied for a 3D scenario. The Q-range can be represented as a function of $x$ and $y$ as

$$
\begin{aligned}
q= & \sqrt{\left(x-x_{1}\right)^{2}+\left(y-y_{1}\right)^{2}} \\
& -\sqrt{\left(x-x_{2}\right)^{2}+\left(y-y_{2}\right)^{2}}-d_{2,1}+d_{2,2} .
\end{aligned}
$$




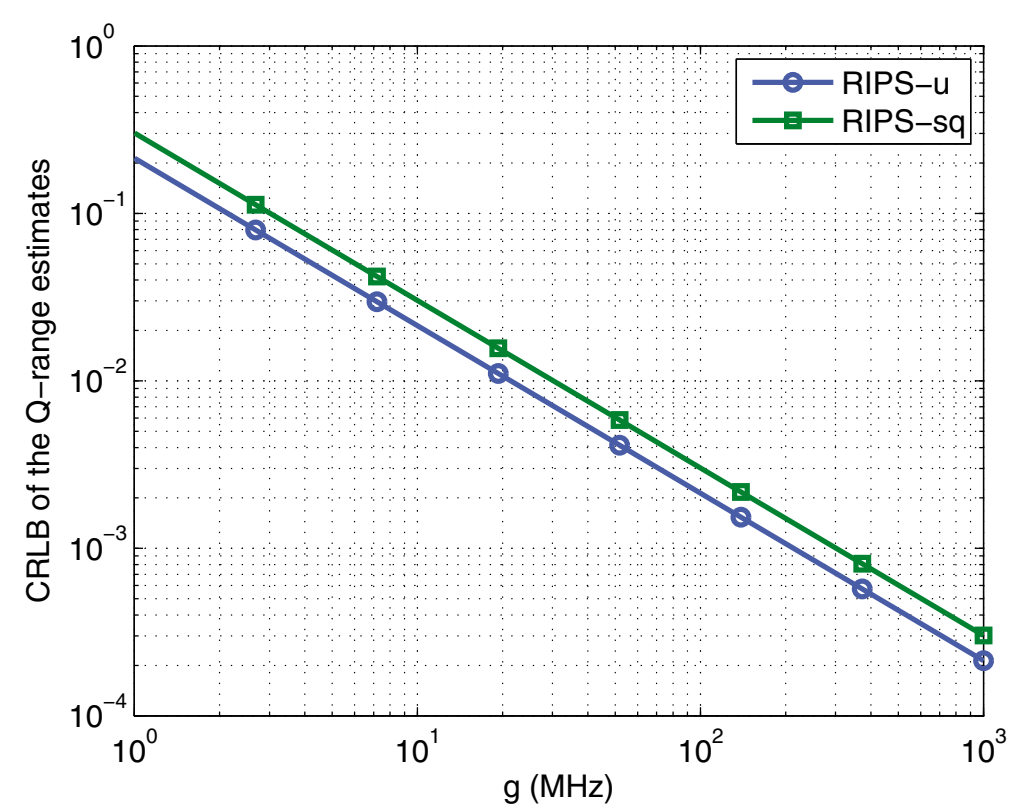

Fig. 4 The CRLB of the Q-range estimates vs. 9

Taking a partial derivative of $q$ with respect to $x$ and $y$, we obtain

$$
\begin{aligned}
& \frac{\partial q}{\partial x}=\frac{x-x_{1}}{d_{1,1}}-\frac{x-x_{2}}{d_{1,2}}, \\
& \frac{\partial q}{\partial y}=\frac{y-y_{1}}{d_{1,1}}-\frac{y-y_{2}}{d_{1,2}} .
\end{aligned}
$$

The RD-based localization requires multiple RD measurements with a common reference node. Suppose we have $M$ anchor nodes, and we fix the first receiver for all the RD measurements. Then, we can achieve up to $M-1$ independent RD measurements. Let us assume that the Q-range estimates are obtained independently using an efficient estimator. In such a case, denoting the $i$ th Q-range measurement as $q_{i}$ for $i=1, \ldots, M-1$

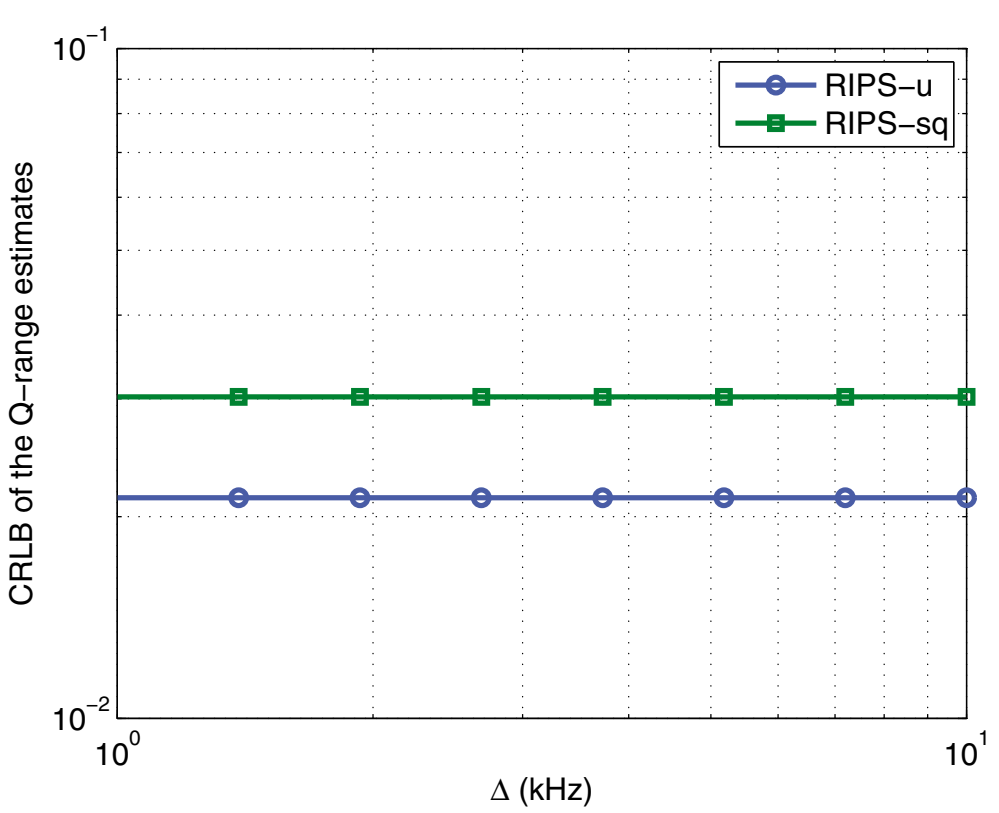

Fig. 5 The CRLB of the Q-range estimates vs. $\Delta$ 


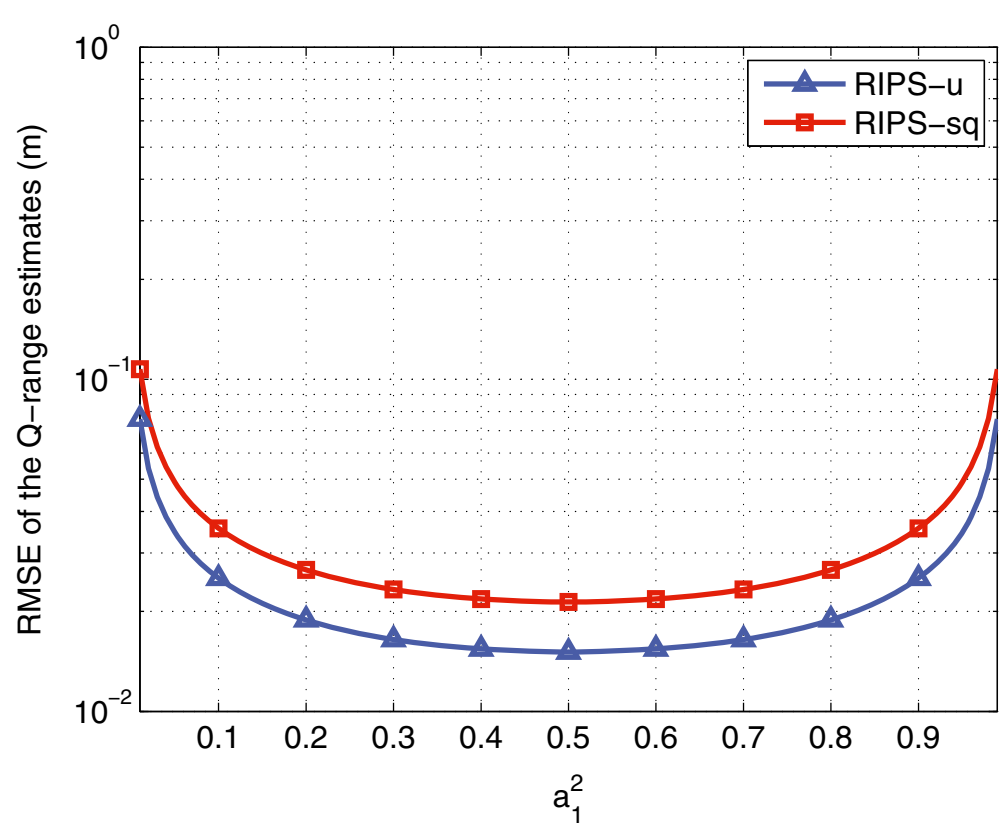

Fig. 6 The CRLB of the Q-range estimates vs. $a_{1}^{2}$

and $\mathbf{q}=\left[q_{1}, q_{2}, \ldots, q_{M-1}\right]^{\mathrm{T}}$, the FIM of $\mathbf{x}$ is given as [31]

$$
\mathbf{J}(\mathbf{x})=\left(\frac{\partial \mathbf{q}}{\partial \mathbf{x}}\right)^{T} \Sigma^{-1}\left(\frac{\partial \mathbf{q}}{\partial \mathbf{x}}\right),
$$

where

$$
\frac{\partial \mathbf{q}}{\partial \mathbf{x}}=\left[\begin{array}{cc}
\frac{\partial q_{1}}{\partial x} & \frac{\partial q_{1}}{\partial y} \\
\vdots & \vdots \\
\frac{\partial q_{M-1}}{\partial x} & \frac{\partial q_{M-1}}{\partial y}
\end{array}\right]
$$

and

$$
\boldsymbol{\Sigma}=\operatorname{diag}\left\{\operatorname{CRLB}\left(q_{1}\right), \ldots, \operatorname{CRLB}\left(q_{M-1}\right)\right\}
$$

Performing matrix multiplications, (39) is rewritten as

$$
\mathbf{J}(\mathbf{x})=\sum_{i=1}^{M-1} \operatorname{CRLB}\left(q_{i}\right)^{-1} \mathbf{G}_{i}
$$

where

$$
\mathbf{G}_{i}=\left[\begin{array}{cc}
\left(\frac{\partial q_{i}}{\partial x}\right)^{2} & \left(\frac{\partial q_{i}}{\partial x}\right)\left(\frac{\partial q_{i}}{\partial y}\right) \\
\left(\frac{\partial q_{i}}{\partial x}\right)\left(\frac{\partial q_{i}}{\partial y}\right) & \left(\frac{\partial q_{i}}{\partial y}\right)^{2}
\end{array}\right],
$$

and CRLB $\left(q_{i}\right)$ in (42) is replaced with the CRLB for the $i$ th Q-range measurement with the RIPS-u in (32) and that with the RIPS-sq in (26), respectively. The CRLBs of $x$ and $y$ are the diagonal entries of the inverse of $\mathbf{J}(\mathbf{x})$ in (42).

As an example, consider the case where five anchor nodes are placed at the center and the corners of a $10 \times 10 \mathrm{~m}^{2}$. A square root of the trace of $\mathbf{J}^{-1}(\mathbf{x})$,
$\sqrt{\operatorname{CRLB}(x)+\operatorname{CRLB}(y)}$, for the RIPS-u and the RIPS-sq at various target node locations are shown in Fig. 7. The SNR is fixed at $30 \mathrm{~dB}$. White circles denote the locations of the anchor nodes. Since the special case defined in (33)-(34) is considered, the CRLBs of the location estimates in two systems are only different by a scalar constant. Therefore, both figures show the same trend that the CRLBs increase as the target node moves away from the origin.

Furthermore, Fig. 8 shows the CRLBs of the location estimates vs. SNR at six different target node locations that are represented by triangle marks in Fig. 7. Solid lines represent the CRLBs corresponding to the RIPS- $u$ and dashed lines correspond to that of the RIPS-sq. At any given location, the RIPS- $u$ has the CRLB $3 \mathrm{~dB}$ lower than that of the RIPS-sq, which is the difference in $\gamma^{(u)}$ and $\gamma^{(\mathrm{sq})}$. Comparing the curves corresponding to positions inside (L1 L3) and outside (L4 L6) the square, we observe that the latter has higher bounds compared to the former, as we have already observed in Fig. 7.

\section{Simulation results}

In this section, we perform Monte Carlo simulations to examine the theoretical bounds derived in Section 4 . The simulation setup and anchor node locations are the same as those presented in Section 4. The target node is randomly placed within a $10 \times 10 \mathrm{~m}^{2}$ at each iteration. Frequencies are set at $g=10 \mathrm{MHz}$ and $f_{o}=9$ $\mathrm{MHz}$, and we simulate over three different $\Delta$ 's, which are $\Delta=1.2,12$, and $120 \mathrm{kHz}$. The sampling frequency is $f_{u}=f_{s}=4 \Delta$, and $N=100$. Here, we use the simplified case where $a_{k}=a$ for $k=1,2$ and $\sigma_{m}^{2}=\sigma^{2}$ for 


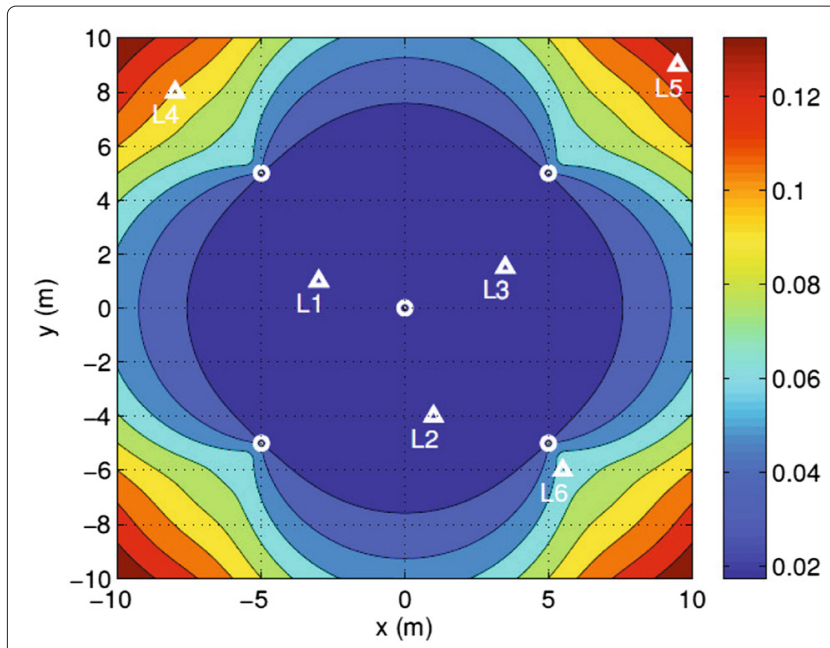

(a)

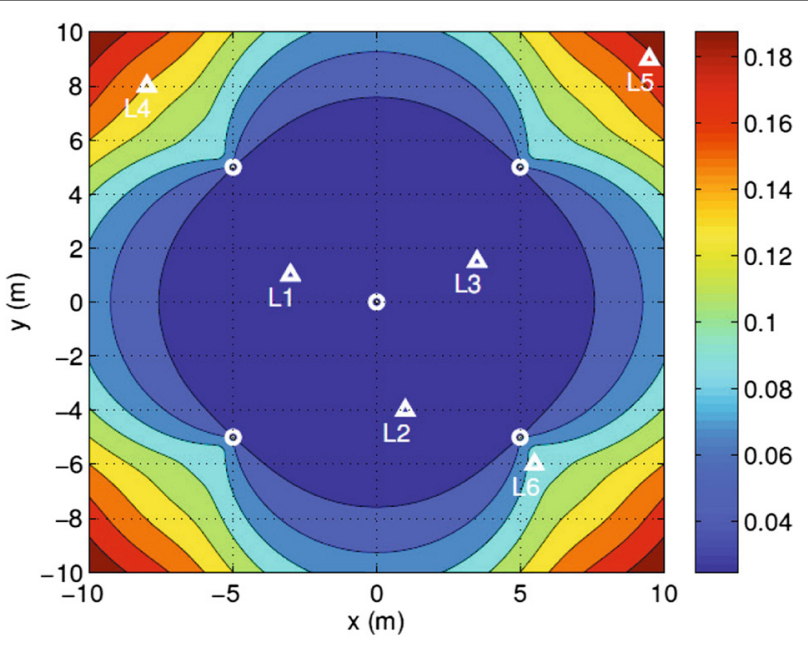

(b)

Fig. 7 The CRLBs of the location estimates of at various locations with $\mathbf{a}$ the RIPS- $u$ and $\mathbf{b}$ the RIPS-sq

$m=1,2$, and the SNR is defined as in Section 4. Since $f_{u}<2\left(f_{k}-f_{o}\right)$, frequencies of the sampled signal are aliased in the RIPS-u. For example, when $\Delta=1.2 \mathrm{kHz}$, frequencies are aliased as $\bmod \left(f_{1}-f_{o}, f_{u}\right)=2.2 \mathrm{kHz}$ and $\bmod \left(f_{2}-f_{o}, f_{u}\right)=1.0 \mathrm{kHz}$. Under this setup, the Q-ranges are always within the resolvable range. Hence, there is no integer ambiguity in both systems.

A root-mean-square error (RMSE) of the Q-range estimates vs. SNR is shown in Fig. 9. Dotted lines represent the CRLBs of the Q-range estimates as given in (34) for the RIPS- $u$ and (33) for the RIPS-sq, and solid and dashed lines correspond to the RMSE curves for the RIPS-sq and the RIPS-u, respectively. Due to the space limitation, only the CRLBs with $\Delta=1.2 \mathrm{kHz}$ are plotted, and both systems attain the CRLBs with this $\Delta$ as shown in Fig. 9. Hence, our assumption that the Q-range estimators are efficient at the derivation of the FIM $\mathbf{J}(\mathbf{x})$ in (42) is valid. As expected from (36), there is approximately a $3-\mathrm{dB}$ gain in the performance of the RIPS-u over that of the RIPSsq. Also, note that there is an error floor in the RMSE curves of the RIPS-sq at high SNR region. This is due to the approximation in the estimator in the RIPS-sq, and the

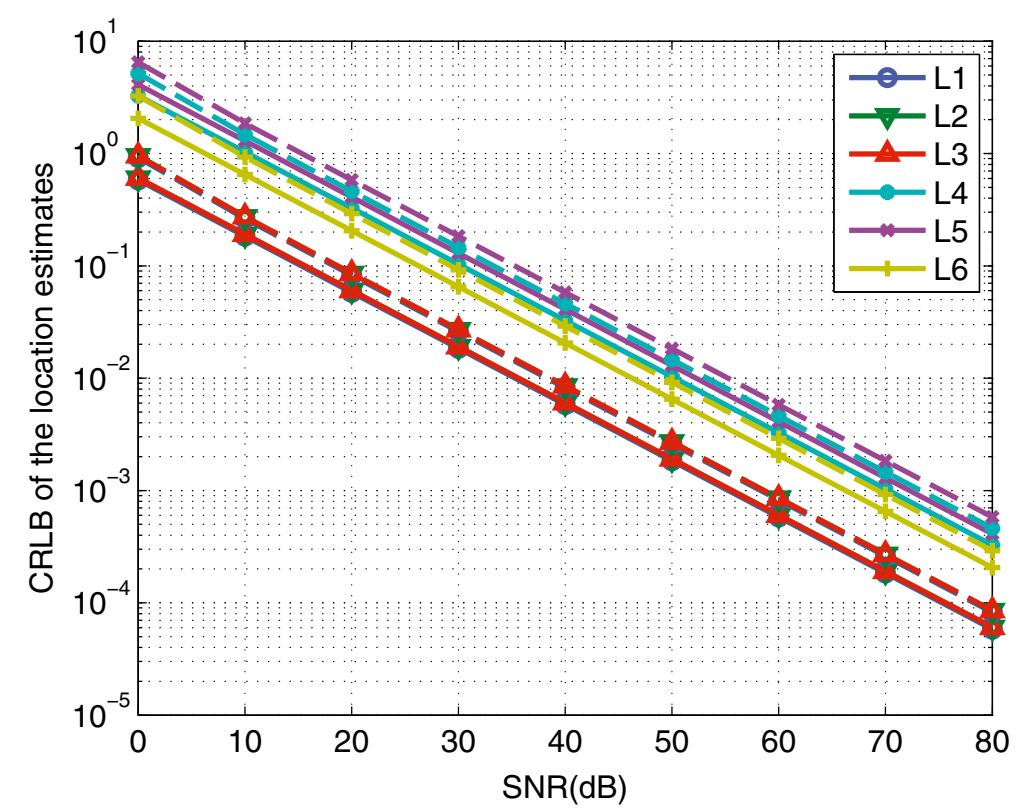

Fig. 8 The CRLBs of the location estimates vs. SNR at specific locations. Solid lines represent the RIPS-u and dashed lines are for the RIPS-sq 


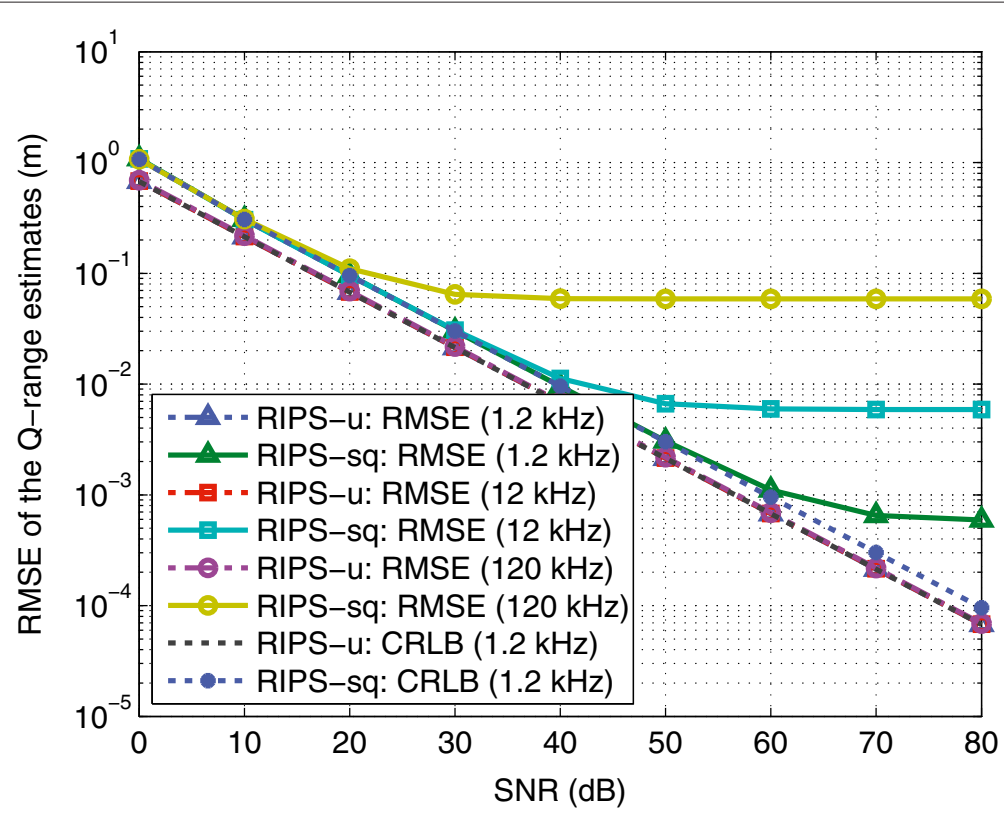

Fig. 9 The RMSE of the Q-range estimates vs. SNR. Dotted lines represents the CRLB curves, and dashed and solid lines correspond to the RMSE curves of the RIPS-u and the RIPS-sq, respectively

error floor increases as $\Delta$ increases since the ratio $\Delta / g$ in the approximated term $\eta$ increases. On the other hand, the performance of the RIPS- $u$ is approximately the same over different $\Delta$ 's. In other words, unlike in the RIPSsq, frequencies are allowed to be further apart without worsening the performance in the RIPS- $u$. Therefore, the RIPS-u has more flexibility in designing parameters than the RIPS-sq.

The RMSEs of the location estimates of two systems vs. SNR are shown in Fig. 10, respectively. We employ the RD-based localization in [28] to estimate the location of the target node. Since the CRLBs depend on the target node position, the average of the CRLBs calculated at each iteration is plotted here. The figure shows the performance gain of the RIPS-u over the RIPS-sq in the location estimation as well.

\section{Experimental evaluation}

To further compare the performance of two systems, we implemented them on the NI USRP transceivers [32].

\subsection{The system model}

We employed four NI USRP 2920 to estimate the Qrange in the RIPS- $u$ and the RIPS-sq. Two USRPs are used as transmitters, and the other two are used as receivers. Although one USRP transceiver is capable of both transmitting and receiving, we use separate devices to allow different spatial arrangements. The internal clock of the
USRP acting as the first receiver is used as the reference clock, and a MIMO cable connects two receivers for synchronization. All four USRPs are connected to a host PC through Ethernet cables. LabVIEW is running at the host PC to control the USRP functions and to perform Q-range estimation. Unfortunately, a square-law device is not equipped in the USRP devices, and thus, the signal is squared after sampling in the RIPS-sq. The lack of a square-law device has advantages as well as shortcomings. The RF part is simplified and non-linearity effect caused by the square-law device is neglected. On the other hand, the sampling rate increases as the raw received signal has to be sampled beyond twice of its maximum frequency. Hence, the parameters have to be chosen carefully to successfully obtain low-frequency differential signals. As all the samples are sent to the host PC for processing, the sampling rate is limited by the host $\mathrm{PC}$ specifications.

Let us denote nodes $A$ and $B$ as transmitters and nodes $\mathrm{C}$ and $\mathrm{D}$ as receivers. The signals given in (1) are transmitted by nodes A and B. At the receivers, the received signal is downconverted by $f_{o}$. Since the bandwidth of the USRP device is $20 \mathrm{MHz}$, frequencies are designed as $f_{k}-f_{o}<20 \mathrm{MHz}$ for $k=1,2$. The downconverted signal is sampled at $f_{s 1}$.

In both systems, the signal is processed accordingly and decimated to the same final sampling rate $f_{s}$ for comparison. In the experiment, the sampling frequency is chosen such that the decimation factor $f_{s 1} / f_{s}$ is an integer. Moreover, the parameters should be designed such that $\bmod \left(\Delta, f_{s}\right) \neq 0$ for the RIPS-u. To fulfill these 


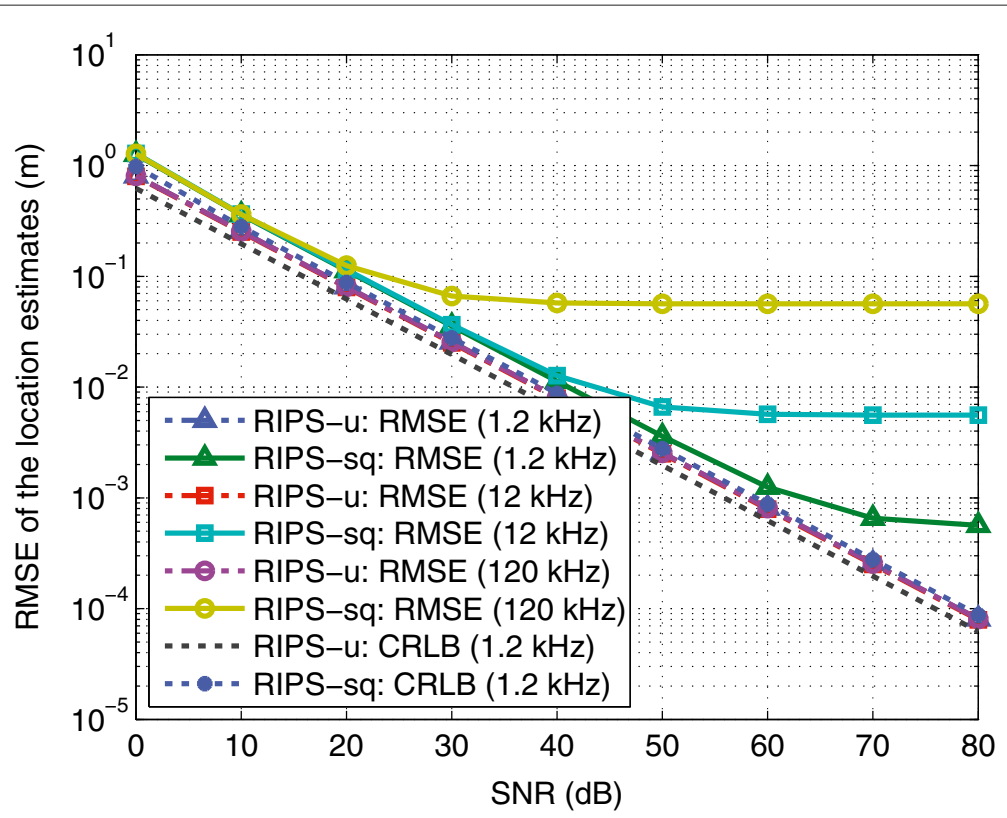

Fig. 10 The RMSE of the location estimates vs. SNR

conditions, we chose $f_{s 1}=4 \mathrm{MHz}$ and $f_{s}=400 \mathrm{kHz}$. As a result, the signal is decimated by the factor of 10 , and $N=10,000$ samples are used to obtain one Q-range estimate.

\subsection{Frequency estimation}

To accurately estimate the phase, the receivers need accurate knowledge of the frequencies. Although the receivers do have knowledge of the designed parameters, the local oscillators at transmitter USRPs and receiver USRPs induce frequency offsets. Hence, the frequencies are estimated from the sampled received signal at the host PC using a frequency detector function from LabVIEW. With the coarse frequency synchronization using a MIMO cable, we have observed the frequency offsets up to $20 \mathrm{~Hz}$.

\subsection{Receiver synchronization}

Recall that the RIPS requires time synchronization between the two receivers. However, the internal clock of the USRP device used as the reference to synchronize two receivers can only yield coarse synchronization. As a result, the phase difference between the two receivers contains two unknown terms: the distance metric and the time offset between the receivers. Hence, we use the true distances, which are physically measured, to calculate the time offset at the beginning of a ranging session. Assuming the time offset between receivers is constant over a period of time, we use this estimated time offset to calibrate the signal prior to the Q-range estimation.

\subsection{Experimental results}

Frequencies used in the experiment are $f_{2}=80 \mathrm{MHz}$, $\Delta=14 \mathrm{kHz}, f_{1}=f_{2}+\Delta$, and $f_{o}=79 \mathrm{MHz}$. The

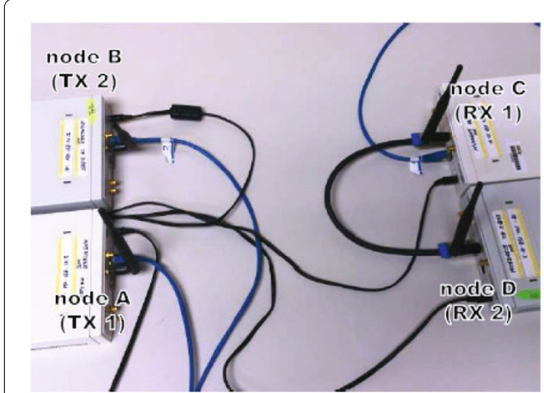

Case 1: $q=4 \mathrm{~cm}$

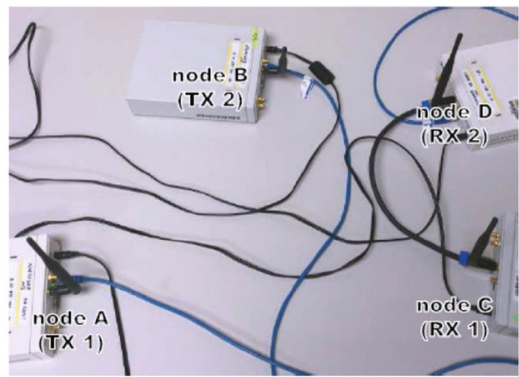

Case 2: $q=20 \mathrm{~cm}$

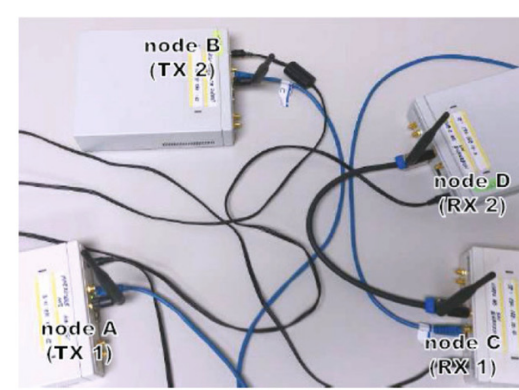

Case 3: $q=-15 \mathrm{~cm}$

Fig. 11 Three experimental setups 


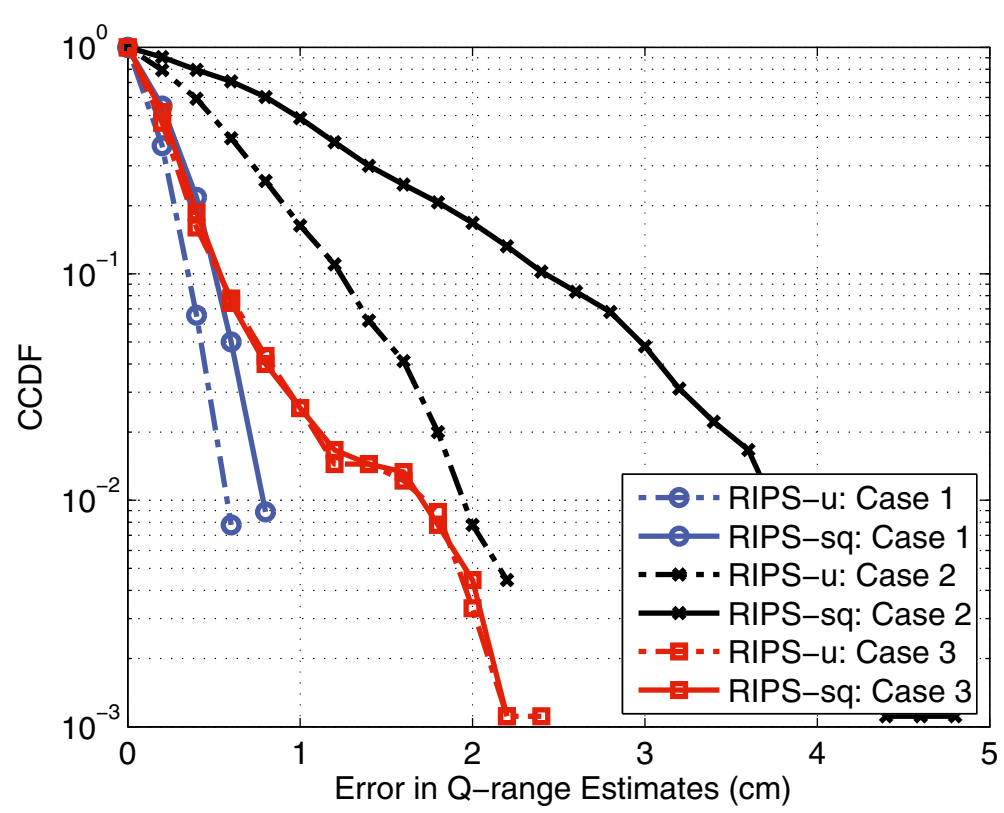

Fig. 12 The complementary cumulative distribution function (CCDF) of the Q-range estimates

received signal is first sampled at $f_{s 1}=4 \mathrm{MHz}$. The signal is squared prior to decimation in the RIPS-sq whereas the signal is directly decimated in the RIPS-u. With these parameters, the approximation error in the RIPS-sq is less than $0.001 \mathrm{~m}$, and the resolvable range for both schemes is $\frac{c}{2 g} \approx \frac{c}{2 f_{k}} \approx 1.875 \mathrm{~m}$. Since the length of the MIMO cable is less than a meter, our experimental setup will not have the Q-range exceeding these bounds.

Four USRPs are placed in three different arrangements, and these setups are shown in Fig. 11. A number below each setup is the true Q-range measured physically. In each setup, the USRP device labeled "node D (RX2)" is connected to the one labeled "node C (RX1)" through the MIMO cable for coarse synchronization. For one Q-range estimate, $N=100,000$ samples are acquired. We assume the receiver and frequency offsets are constant over a period of time required to obtain 100 Q-range estimates, and the offsets are recalculated every $100 \mathrm{Q}$-range estimates. Repeating this process for nine times, 900 Q-range estimates are achieved.

Complementary cumulative distribution function (CCDF) of the Q-range estimation errors for three setups are presented in Fig. 12. Although we assumed that the time and frequency offsets are constant over 100 Q-range estimates, we observed the random fluctuations in frequency offsets. The frequency offset causes the unknown bias in the estimated phase, and the same frequency offsets produce the different bias in two systems. As a result, the frequency offset instability caused the difference in the performance among three cases. Yet, the performance gain of the RIPS-u over the RIPS-sq is noticeable, especially in case 2 . Furthermore, the RIPS- $u$ yields smaller standard deviation compared to the RIPSsq as shown in Table 1. Hence, the experimental results confirm that the RIPS-u has better performance than the RIPS-sq.

\section{Conclusions}

In this paper, we investigated the performance of the RIPS using the receiver with square-law devices (RIPS-sq) and with undersampling techniques (RIPS-u) through theoretical and experimental analyses. In the theoretical analysis, we computed Cramér-Rao lower bounds (CRLBs) for the Q-range and location estimates for both systems and showed that the RIPS-u has a lower CRLB compared to the RIPS-sq. For the experimental analysis, we implemented two systems onto the USRP devices and confirmed the accuracy of the RIPS-u over the RIPS-sq.

Table 1 Medians and standard deviations of the estimated Q-ranges

\begin{tabular}{lllllll}
\hline & \multicolumn{2}{c}{ RIPS-u } & & \multicolumn{2}{c}{ RIPS-sq } \\
\cline { 2 - 3 } \cline { 6 - 7 } & $\begin{array}{l}\text { Median } \\
(\mathrm{cm})\end{array}$ & $\begin{array}{l}\text { Std. dev. } \\
(\mathrm{cm})\end{array}$ & & $\begin{array}{l}\text { Median } \\
(\mathrm{cm})\end{array}$ & $\begin{array}{l}\text { Std. dev. } \\
(\mathrm{cm})\end{array}$ \\
\hline Case 1 $(4 \mathrm{~cm})$ & 4.0072 & 0.2207 & & 4.0056 & 0.3155 \\
Case 2 $(20 \mathrm{~cm})$ & 19.8753 & 0.7346 & & 19.8753 & 1.4525 \\
Case 3 $(-15 \mathrm{~cm})$ & -14.9520 & 0.3737 & & -14.9673 & 0.3850 \\
\hline
\end{tabular}




\section{Competing interests}

The authors declare that they have no competing interests.

\section{Author details}

${ }^{1}$ School of Electrical and Computer Engineering, Georgia Institute of Technology, 30332-0250 Atlanta, GA, USA. ${ }^{2}$ Department of Automation, Shanghai Jiao Tong University, 200240 Shanghai, China.

\section{Received: 9 April 2015 Accepted: 27 August 2015}

Published online: 17 September 2015

\section{References}

1. IF Akyildiz, W Su, Y Sankarasubramaniam, E Cayirci, A survey on sensor networks. IEEE Commun. Mag. 40(8), 102-114 (2002)

2. N Patwari, JN Ash, S Kyperountas, AO Hero, RL Moses, NS Correal, Locating the nodes: cooperative localization in wireless sensor networks. IEEE Signal Process. Mag. 22(4), 54-69 (2005)

3. G Sun, J Chen, W Guo, KJR Liu, Signal processing techniques in network-aided positioning: a survey of state-of-the-art positioning designs. IEEE Signal Process. Mag. 22(4), 12-23 (2005)

4. BM Sadler, Fundamentals of energy-constrained sensor network systems. IEEE Aerosp. Electron. Syst. Mag. 20(8), 17-35 (2005)

5. S Gezici, Z Tian, GB Giannakis, H Kobayashi, AF Molisch, HV Poor, Z Sahinoglu, Localization via ultra-wideband radios: a look at positioning aspects for future sensor networks. IEEE Signal Process. Mag. 22(4), 70-84 (2005)

6. D Dardari, A Conti, U Ferner, A Giorgetti, MZ Win, Ranging with ultrawide bandwidth signals in multipath environments. Proc. IEEE. 97(2), 404-426 (2009)

7. M Maróti, B Kusý, G Balogh, P Völgyesi, A Nádas, K Molnár, S Dóra, A Lédeczi, in Proc. ACM SenSys. Radio interferometric geolocation (ACM San Diego, CA, 2005), pp. 1-12

8. N Patwari, AO Hero III, in Proc. EmNets. Indirect radio interferometric localization via pairwise distances (EmNets Cambridge, MA, 2006)

9. XWang, B Moran, M Brazil, in Proc. IEEE ICON. Hyperbolic positioning using RIPS measurements for wireless sensor networks (IEEE Adelaide, SA, Australia, 2007), pp. 425-430

10. M Shinotsuka, Y Wang, X Ma, GT Zhou, in Proc. IEEE MILCOM. Designing radio interferometric positioning system employing undersampling techniques (IEEE Baltimore, MD, 2014), pp. 312-316

11. B Kusý, A Lédeczi, X Koutsoukos, in Proc. ACM SenSys. Tracking mobile nodes using RF doppler shifts (ACM Sydney, Australia, 2007), pp. 29-42

12. B Kusý, G Balogh, J Sallai, A Lédeczi, M Maróti, in Proc. EWSN. Intrack: high precision tracking of mobile sensor nodes (EWSN Delft, The Netherlands, 2007), pp. 51-66

13. I Amundson, X Koutsoukos, J Sallai, in Proc. ACM MELT. Mobile sensor localization and navigation using RF doppler shifts (ACM San Francisco, CA, 2008), pp. 97-102

14. TI CC1000. Texas Instruments. http://www.ti.com/product/cc1000 Accessed 10 Sept 2015

15. BJ Dil, PJM Havinga, in Proc. IEEE MASS. A feasibility study of RIP using 2.4 GHhz 802.15.4 radios (IEEE San Francisco, CA, 2010), pp. 690-696

16. BJ Dil, PJM Havinga, in Proc. ACM SenSys. Stochastic radio interferometric positioning in the $2.4 \mathrm{GHz}$ range (ACM Seattle, WA, 2011), pp. 108-120

17. TI CC2430. Texas Instruments. http://www.ti.com/product/cc2430. Accessed 10 Sept 2015

18. W Zhang, Q Yin, X Feng, W Wang, in Proc. IEEE VTC. Distributed TDOA estimation for wireless sensor networks based on frequency-hopping in multipath environment (IEEE Taipei, Taiwan, 2010), pp. 1-5

19. X Li, K Pahlavan, Super-resolution TOA estimation with diversity for indoor geolocation. IEEE Trans. Wireless Commun. 3(1), 224-234 (2004)

20. Y Wang, X Ma, C Chen, X Guan, Designing dual-tone radio interferometric positioning systems. IEEE Trans. Signal Process. 63(6), 1351-1365 (2015)

21. Y Wang, L Li, X Ma, M Shinotsuka, C Chen, X Guan, Dual-tone radio interferometric positioning systems using undersampling techniques. IEEE Signal Process. Lett. 21(11), 1311-1315 (2014)

22. M Shinotsuka, Y Wang, X Ma, GT Zhou, in Proc. Asilomar Conf. on Signals, Systems, and Computers. Designing radio interferometric positioning systems for indoor localizations in millimeter wave bands (IEEE Pacific Grove, CA, 2014)
23. W Li, X Wang, B Moran, in Proc. FUSION. Resolving RIPS measurement ambiguity in maximum likelihood estimation (IEEE Chicago, IL, 2011), pp. 1-7

24. C Wang, Q Yin, W Wang, An efficient ranging method based on Chinese remainder theorem for RIPS measurement. Sci. China Inf. Sci. 53(6), 1233-1241 (2010)

25. C Wang, Q Yin, H Chen, Robust Chinese remainder theorem ranging method based on dual-frequency measurements. IEEE Trans. Veh. Technol. 60(8), 4094-4099 (2011)

26. W Li, X Wang, X Wang, B Moran, Distance estimation using wrapped phase measurements in noise. IEEE Trans. Signal Process. 61(7), 1676-1688 (2013)

27. G Xu, L Zhang, Y Wang, Y-H Hu, in Proc. IEA. The influence of the carrier frequency inaccuracy and multipath effects on radio interferometric positioning system, vol. 154 (London: Springer Chongqing, China, 2012), pp. 847-854

28. P Stoica, J Li, Lecture notes - source localization from range-difference measurements. IEEE Signal Process. Mag. 23(6), 63-66 (2006)

29. Y Wang, G Leus, Reference-free time-based localization for an asynchronous target. EURASIP J. Adv. Sig. Process (2012). doi:10.1186/1687-6180-2012-19

30. S Kay, Fundamentals of Statistical Signal Processing: Estimation Theory (Prentice-Hall, Inc., Eaglewood Cliffs, NJ, 1993)

31. R Kaune, J Horst, W Koch, in Proc. FUSION. Accuracy analysis for TDOA localization in sensor networks (IEEE Chicago, IL, 2011), pp. 1648-1654

32. NI Universal Software Radio Peripheral (USRP). National Instruments. http://www.ni.com/usrp/. Accessed 10 Sept 2015

\section{Submit your manuscript to a SpringerOpen ${ }^{\circ}$ journal and benefit from:}

- Convenient online submission

- Rigorous peer review

- Immediate publication on acceptance

- Open access: articles freely available online

- High visibility within the field

- Retaining the copyright to your article

Submit your next manuscript at $>$ springeropen.com 